\title{
Systems Transdisciplinary Approach in the General Classification of Scientific Approaches
}

\author{
Vladimir Mokiy, PhD \\ Institute of Transdisciplinary Technologies, Russia
}

Doi:10.19044/esj.2019.v15n19p247 URL:http://dx.doi.org/10.19044/esj.2019.v15n19p247

\begin{abstract}
The development of systems thinking and systems worldview needs new types of system approaches. New types of systems approaches should find their place in the appropriate classification of scientific approaches. The article attempts to substantiate the natural connection of classical scientific approaches with their system analogues. This relationship is manifested in the General classification of scientific approaches from the mono-disciplinary approach to the systems transdisciplinary approach. The definitions of the approaches in such a classification are distinguished by identification features and functional features. Classification allows you to consciously use these definitions within a single semantic field of scientific knowledge. The streamlining of scientific approaches within the framework of such a classification makes it possible to give a rationale for a wide range of interdisciplinary interactions in modern science.
\end{abstract}

Keywords: Systems worldview; Systems thinking; Classification of systems approaches; Russian School of Transdisciplinarity

\section{Introduction}

The hopes for the appearance of new scientific approaches that allow working with the whole complex of social, economic and environmental problems of modern society are associated with the development of a systems worldview. In the light of the importance of worldviews to systems science and its applications, it is surprising that systems science does not have a canonical model of the structure and dynamics of worldviews, and hence does not provide for a consistent way of working with worldviews across systems theories and methodologies (Rousseau \& Billingham, 2018). Such a method can be found in the logical connections between classical scientific approaches and their systems analogues, between the classical and systems worldviews within the framework of the general classification of scientific approaches. 


\section{Classification of the scientific approaches}

The scientific community is trying to overcome the mono-disciplinary fragmentation of science, caused by a variety of local pictures of the world. In this regard it adopts a certain system of rules, norms and standards. Such a system is called scientific rationality. Different scientific communities may pursue the same goal. At the same time, they can interpret the meaning of scientific rationality in different ways. In this case, to each other, they may look irrational. In such a situation, researchers have two obvious choices. In the first case, they must defend their point of view. In the second case, they will be forced to trust the opinion of scientific authorities and take on faith a certain sense of scientific rationality (Porus, 1995).

In the history of the development of various forms of human activity, there are cases when a successful choice was unrelated to obvious solutions. This choice came down to the use of new paradigms, new pictures of the world, new philosophical decisions. As a rule, this choice is possible within the framework of periodically arising stages of the integration of scientific knowledge. The increasing level of knowledge integration contributed to the emergence of inter-disciplinary, multi-disciplinary and trans-disciplinary approaches in science. At the same time, the integral picture of the world of these approaches, with its practical application, continued to "fall apart" into mono-disciplinary knowledge. And the analysis of this knowledge still required a methodology of mono-disciplinary approaches. An alternative to this circumstance was a systems worldview. In the systems worldview, the world is associated with the abstract image of the system. Therefore, within the framework of a systems worldview and a systems approach, there is hope for the integration of various types of scientific rationality, contributing to the emergence of a rationality of a single science.

In a single science, it does not matter what place a person takes in the world. It is important that a person agrees with the existence of an objective categorical imperative. I. Kant's categorical imperative makes it possible to judge the morality of a person's actions. The objective categorical imperative of a single science makes it possible to judge the form of manifestation of the universal order, which makes the world around us one. The objective categorical imperative, as an objective "sphere of obligation", manifests itself in the interaction of all objects at all levels of reality in a single space, information and time. Thereby, within the framework of an objective categorical imperative, imperatives of all types of human activity receive objective restrictive conditions.

It should be noted that the idea of a unified science is disputed by some scholars. However, the long-term ascent of the scientific community to a single science can be fixed within the framework of the general classification of scientific approaches. Such a classification is able to demonstrate a 
consistent expansion of the boundaries of the classical scientific worldview in the direction: from the linear logic of the classical science approaches to the context logic of the systems approaches.

\subsection{Definitions of the scientific approaches}

Existing scientific approaches to the knowledge of the world that use linear logic can be divided into two main groups. Approaches of the first group provide the formation and development of a scientific worldview. The approaches of the first group include mono-disciplinary approaches of academic scientific disciplines. Approaches of the second group determine the expansion of the horizons of the formed scientific worldview. The second group of approaches includes interdisciplinary approaches. Inter-disciplinary approaches include: inter-disciplinary, multi-disciplinary and transdisciplinary approaches.

Mono-disciplinary approaches have shaped the scientific worldview. The linear logic of mono-disciplinary approaches corresponds to a certain process. In the course of this process, knowledge is successively added to the objects of research, to a mono-disciplinary image of an object, and then to a local picture of the world. Therefore, mono-disciplinary approaches are designed to perform several basic actions: obtaining the maximum amount of knowledge about the object; highlighting the subjects of the study in the object; the formation of theoretical principles and techniques of monodisciplinary research.

The increasing complexity of the mono-disciplinary image of the study object creates the conditions for the emergence of inter-disciplinary interactions in science. Such interactions are carried out in the framework of inter-disciplinary, multi-disciplinary and trans-disciplinary approaches. As applied to the general classification of scientific approaches, their contextual definitions will be as follows:

An inter-disciplinary approach is a way to expand the scientific worldview in the direction of enriching the knowledge, methodology and language of one scientific discipline at the expense of knowledge, methodology and language of another scientific discipline. The presence of similar subject areas allows you to use the methodology of one discipline to solve problems of another discipline. The main identification of interdisciplinary approaches is the establishment of subordination between the interacting disciplines. The "leading" discipline shapes the issues and objectives of inter-disciplinary research. From the position of the leading discipline, the final interpretation of the results of inter-disciplinary research is carried out. "Subordinate" discipline provides for inter-disciplinary research only its own methodological apparatus (Mokiy, 2009). 
A multi-disciplinary approach is a way of expanding the scientific worldview in the direction of a holistic image of the studied object. Multidisciplinary approaches allow you to search for a combination of different subject areas that are important for the object under study. Within the framework of multi-disciplinary approaches, the opportunity has appeared to study an object with the methodology of different disciplinary and interdisciplinary approaches. Multi-disciplinary research is defined as research conducted in several disciplines and pursuing several independent goals. At the same time, they are united by a common target context (Petts, Owens, Bulkeley, 2008). The main identification features of multi-disciplinary approaches are: the presence of a target research context that does not belong to any single discipline, as well as consensus and compromises, resulting in an intersubjective (accepted by most people) research result.

The trans-disciplinary approach is a way of expanding the scientific worldview, which consists in examining an object outside of any single scientific discipline. Trans-disciplinary approaches have arisen due to the need to study objects of increased complexity without their separation from the environment. In the absence of strict identification signs, the trans-disciplinary approach today is perceived as a special type of scientific research that goes through, across the boundaries of many disciplines, going beyond them, which follows from the very nature of the prefix "trans" (Knyazeva, 2011).

\section{Classification of the system approaches}

The arsenal of modern cognitive tools was created mainly by classical science. It has an analytical nature and is unsuitable for the analysis of integrity, hierarchy and complex organization. At the same time, systems thinking can be developed by modifying the existing cognitive means of classical science. At present, to describe systems thinking, systems research methods, we are forced to use non-systems in its essence concepts, concepts and methods. This, ultimately, is the general basis for the emergence of systemic paradoxical situations (Sadovcky, 1974). It is logical that such a modification of scientific approaches will consist of system approaches similar to those of classical science. It is important to make a decision on how to determine the solutions of the worldviews. As Martin Hall explained, the power of systems methodologies derives from their taking account of worldviews, because worldviews create the context both for adequate modelling of problems and for appropriate selection of solutions (Hall, 1995). Therefore, system approaches will interpret the term "system" in different ways. Such approaches are: systems disciplinary, systems interdisciplinary, systems multidisciplinary and systems transdisciplinary approaches. 


\subsection{Definitions of the system approaches}

The definitions of systems approaches differ in the way of correctly selecting and modeling an object in the image of the system.

The systems disciplinary approach is a method of correct highlighting and modeling an object in the image of a local mono-disciplinary system. Such an image of the system allows one to apply the corresponding systemdisciplinary methodology to the study of the object. Systems disciplinary approaches demonstrate a special way of integrating disciplinary knowledge. Not all existing knowledge is included in the integration process. Such knowledge is specifically selected by the researcher according to certain criteria when modeling an object as a system. In this case, systems disciplinary approaches, in essence, are a form of perception of the methods and principles of classical systems research. This form of perception is used by specialists of specific scientific disciplines working in the field of specific scientific knowledge. The development of systems disciplinary approaches contributed to the emergence in the environment of diverse scientific knowledge of empirical systems scientific disciplines (Systemics). Such disciplines are: systems psychology, systems biology, systems psychiatry, etc.

The systems interdisciplinary approach is a method of correct $s$ highlighting and modeling of an object in the image of a local interdisciplinary system. This approach allows one to apply complementary systems disciplinary methodologies to the study of the object. Systems interdisciplinary approaches demonstrate a way to integrate the disciplinary knowledge of similar subject areas of objects within the framework of modeling their relationships as a system. Such integration complicates the logic and methodology of interdisciplinary systems research. It contributes to the enrichment of the language of empirical systems scientific disciplines (Systemics). In their highest form, system-interdisciplinary approaches are able to form the so-called interdisciplinary systems paradigms.

Systems disciplinary and systems interdisciplinary approaches are more dependent on the empirical description of system research procedures. The success of these approaches determines the modeling of the object in the image of the system, supported by its strict mathematical expression. The principle of simplifying the image of an object during its systems modeling is transferred to the formation of local pictures of the world. Subjective relief of the world picture from non-essential characteristics can accidentally exclude from the field of view of the researcher those characteristics that, under certain conditions, can play the role of factors determining the development of an object.

The following two approaches from the classification of systems approaches allow one to eliminate this circumstance. These approaches include: systems multidisciplinary and systems transdisciplinary approaches. 
These approaches are more dependent on the existing general philosophical concepts, on the image of the world picture, which influence the content of the ontological and epistemological aspects of the study of systems. Important for the development of these types of system approaches are:

- heuristic, systematizing and ideological functions of the world picture;

- concepts of space, time and information, as philosophical categories that are directly related to fundamental objects (world, universe);

- the definition of the concept of a "categorical imperative" in a subjective and objective context, determining, respectively, the integrity or unity of the object, as a system.

The systems multidisciplinary approach is a way to correctly isolate and model a complex object as a holistic multidisciplinary system. The image of a holistic multidisciplinary system is associated with a set of objects that are combined to achieve a specific goal. To discover the order that determines the integrity of an object as a system, this approach uses an appropriate set of systems disciplinary methods. The whole world consists of parts. These parts outside the entire are of independent sense. The concept and the view of the whole world do not forbid the existence of other entire worlds, of other entire objects. For this reason, it is necessary to justify the completeness of a set of object parts as a system in each specific case within the framework of systems multidisciplinary approach, and then to identify or subjectively establish the order that determines the interaction of these parts.

The philosophical basis of the systems multidisciplinary approach is holism. Holism, in a broad sense, is a position in philosophy and in science regarding the problem of the relationship between part and whole. This position is based on the qualitative originality of the whole in relation to its parts. In ontology, holism is based on the principle: the whole is always more than the sum of its parts. The epistemological principle of holism says: the knowledge of the whole must precede the knowledge of its parts. In a narrower sense, holism is understood as the "philosophy of integrity" developed by the South African philosopher J. Smuts, who in 1926 coined the term "holism" (Nikiforov, 2010). Integrity implies a unique combination and consistency of parts. This circumstance sets the vector of search and description of a hypothetical (subjective) imperative in a specific scientific study of a specific set of objects.

The systems transdisciplinary approach is a way to correctly isolate and model a complex object as a single transdisciplinary system. At the same time, a set of objects pursuing a common goal is associated with a functional ensemble of objects. The image of the transdisciplinary system in this case is associated with the general order, which determines the unity of the functional ensemble of objects. This approach allows the use of a special transdisciplinary concept, philosophical basis and methodology in the study. 
The united world is the one world. Any objects at all levels of the reality of a one world are its natural elements/fragments. Therefore, the main condition for the existence of a one world is the existence of a universal order in it. From the name it follows that this objective order must manifest itself everywhere: in every element/fragment of this world, in every interaction of these elements/fragments at every level of reality. As a result, the same order should ensure the achievement of activity goals and results of all these elements/fragments and synchronize these goals and results. For this reason, a single world is a One Orderly Medium.

The major attribute of this One Orderly Medium is the potency, which is naturally present in it. Potency is the prospective futurity of the One Orderly, her latent force. Medium. Within the framework of the unicentrism concept, the definitions of these philosophical categories are as follows:

Space - as a form of existence of potency of One Orderly Medium;

Information - as a form of development of potency of One Orderly Medium; Time - as a form of transformation of potency of One Orderly Medium.

The universal order plays the role of a transdisciplinary system in relation to the forms of potentiality of a single world. This particular universal order manifests in the forms themselves, in the interaction of these forms, as well as determines their unity.

The philosophical basis of the systems transdisciplinary approach is "centrism of unity" (unicentrism). In a broad sense, unicentrism is a position in philosophy and in science on the problem of the correlation between the single and its fragments. This position is based on the isomorphism of the universal order of the structure of fragments of space, attributes of information and periods of time that determine the one and only of the world (see figures $1 \mathrm{a} ; 1 \mathrm{~b} ; 2 ; 3$ ). All models have a similar structure fragments of space, attributes of information, periods of time. In this case, the image of the transdisciplinary system is revealed by means of models of spatial, informational and temporal "units of order". Methodological features of "unit of order" models follow from their definitions. Systems transdisciplinary model of spatial unit of order is a logically complete structure of space fragments in the transdisciplinary system, which allows substantiation of physical boundaries, within which the original potency of an object and a functional ensemble of objects exist. Systems transdisciplinary model of informational unit of order is a logically complete sequence of attributes of complete information in the transdisciplinary system, which allows the substantiation of logical boundaries of expression of the original potency of an object and a functional ensemble of objects exist. Systems transdisciplinary model of temporal unit of order is a logically complete sequence of time periods in the system, which allows the substantiation of duration of transformation of the original potency of object and a functional ensemble of objects exist. (Mokiy \& Lukyanova, 2017). 


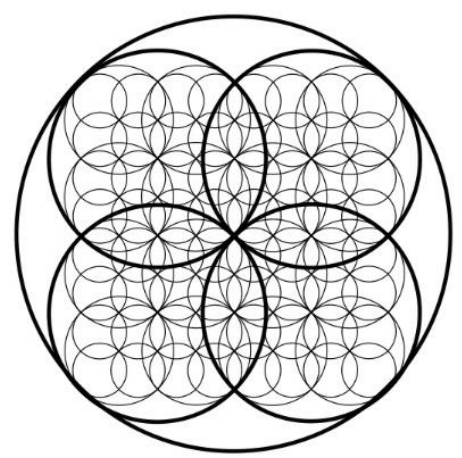

Figure 1a: Systems transdisciplinary model of spatial unit of order [6] (p. 89)

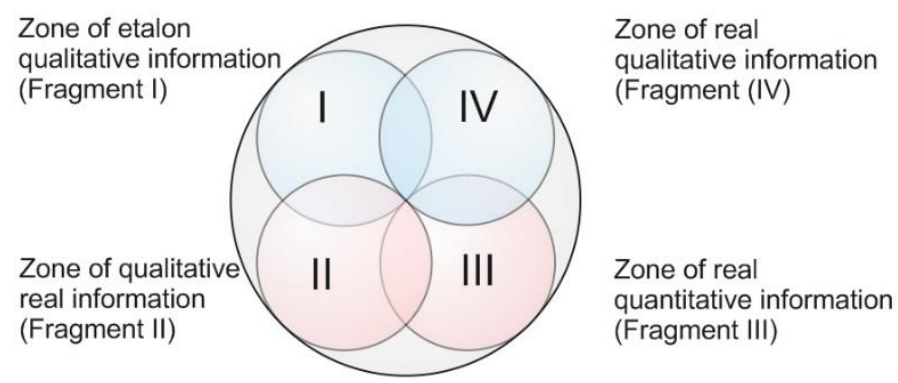

Figure 1b: Scheme of zones of major functional predisposition of spatial unit of order [6] (p. 90)

\begin{tabular}{|l|l|l|l|}
\hline \multicolumn{4}{|c|}{ Complete information } \\
\hline \multicolumn{2}{|c|}{ Information of quantitative type } & \multicolumn{2}{|l|}{ Information of qualitative type } \\
\hline $\begin{array}{l}\text { Information } \\
\text { of quantitative- } \\
\text { quantitative } \\
\text { type }\end{array}$ & $\begin{array}{l}\text { Information } \\
\text { of quantitative- } \\
\text { qualitative } \\
\text { type }\end{array}$ & $\begin{array}{l}\text { Information } \\
\text { of qualitative- } \\
\text { quantitative } \\
\text { type }\end{array}$ & $\begin{array}{l}\text { Information } \\
\text { of qualitative- } \\
\text { qualitative } \\
\text { type }\end{array}$ \\
\hline & Attributes of complete information & \\
\hline
\end{tabular}

Figure 2: Systems transdisciplinary model of information unit of order [6] (p. 69) 


\begin{tabular}{|c|c|c|c|c|c|c|c|}
\hline \multicolumn{8}{|c|}{ Model of multiplex } \\
\hline \multicolumn{8}{|c|}{ Basic waves } \\
\hline \multicolumn{4}{|c|}{ Setting wave } & \multicolumn{4}{|c|}{ Setting wave } \\
\hline \multicolumn{2}{|c|}{ Calibration wave } & \multicolumn{2}{|c|}{ Calibration wave } & \multicolumn{2}{|c|}{ Calibration wave } & \multicolumn{2}{|c|}{ Calibration wave } \\
\hline $\begin{array}{l}\text { Structural } \\
\text { wave }\end{array}$ & $\begin{array}{l}\text { Structural } \\
\text { wave }\end{array}$ & $\begin{array}{l}\text { Structural } \\
\text { wave }\end{array}$ & $\begin{array}{l}\text { Structural } \\
\text { wave }\end{array}$ & $\begin{array}{l}\text { Structural } \\
\text { wave }\end{array}$ & $\begin{array}{l}\text { Structural } \\
\text { wave }\end{array}$ & $\begin{array}{l}\text { Structural } \\
\text { wave }\end{array}$ & $\begin{array}{l}\text { Structural } \\
\text { wave }\end{array}$ \\
\hline
\end{tabular}

Model of dynamic transformation of potency of principles and values of liberal society

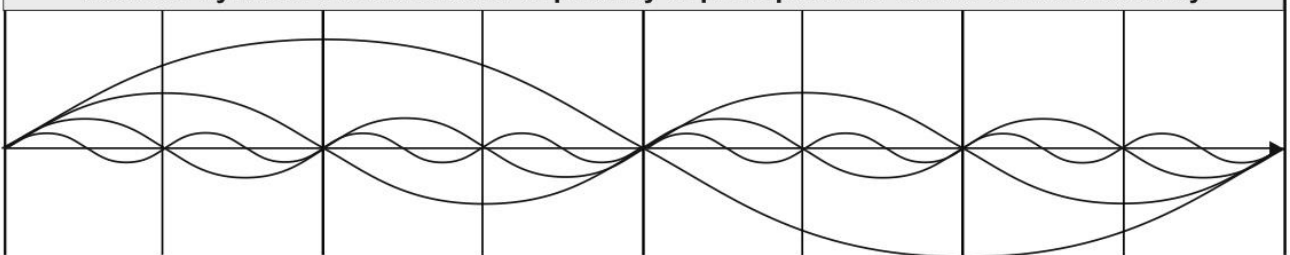

Figure 3: Systems transdisciplinary model of temporal unit of order [6] (p. 125)

In ontology, unicentrism is based on the principle: the one and only world is represented as the sum of ordered fragments of space, attributes of information and periods of time that determine the unity of goals and results of the development of phenomena and processes of reality. The epistemological principle of unicentrism says: the knowledge of the one and only world must be preceded by the selection of appropriate models of spatial, informational, and temporal units of the universal order. In a narrower sense, the unicentrism is understood as the "philosophy of unity" developed by the Russian philosopher Vladimir Mokiy. He also in 2010 introduced the term "unicentrism".

The status of a single object indicates the need for directive placement of mono-disciplinary knowledge in accordance with the structure predetermined by the general order for fragments of space, attributes of information and time periods. Therefore, the order determining unity is not revealed in the course of systems transdisciplinary research of a complex object. It is not formed subjectively, as is done in the systems multidisciplinary approach. It is postulated through systems transdisciplinary models of spatial, informational, and temporal units of order. Thanks to these models, the researcher operates not only with available knowledge of similar and dissimilar subject areas, their interaction. He initially determines their number and types, as well as the nature and consequences of such interaction. Thus, it initially forms the content of an objective categorical imperative, which can be spoken of as a system analogue of the D.I. Mendeleev's periodic table. The new 'periodic table' might enable the discovery of hitherto unknown and unsuspected kinds of systemic structures, behaviours or capacities existing in nature, opening the way for more effective systems methodologies (Rousseau, Billingham, Wilby, Blachfellner, 2016a). It would be possible to ensure that it was possible to establish a system that would allow it to be in accordance with the nature of the system. As such, the systems transdisciplinary approach can 
play the role of a meta-science based on a single set of concepts, a metalanguage and a systems methodology.

\section{Conclusion}

L. Bertalanffy believed that "the General Theory of Systems" is an expression of significant changes in the conceptual picture of the world that emerged in the twentieth century. The peculiarity of the new picture of the world he/she described was "organized complexity". The transformation of organized complexity into a subject of scientific research resulted in the formulation of new cognitive tasks. These tasks, according to L. Bertalanffy, are: 1) the formulation of general principles and laws of systems, regardless of their special type, the nature of their constituent elements and the relations between them; 2) the establishment by analysis of biological, social and behavioral objects, as systems of a special type, of exact and strict laws in the non-physical fields of knowledge; 3) creation of the basis for the synthesis of modern scientific knowledge as a result of revealing the isomorphism of laws relating to various spheres of reality (Bertalanffy, 1967).

For 60 years since its publication, the idea of a General Theory of Systems has been repeatedly criticized. For example, the universality status of the system in the framework of the General Theory of Systems of L. Bertalanffy is rightly criticized, since this statement ignores the main problem of Systemology, the disclosure of the system-forming factor (Anokhin P. 1975). Dealing with the development of a systems worldview within the framework of the classification of systems approaches presented above, it can be argued that the solution to these problems is impossible without a corresponding philosophical basis. A certain philosophical basis treats the system-forming factor in different ways. The role of the backbone factor in the philosophical principle of holism and in the concept of a systems multidisciplinary approach is played by the specific result of the system, as a set of objects (parts). The lack of results can destroy the system. It is able to activate the mechanisms for selecting new components (parts) and the order of their interaction. In its turn, the role of the system-forming factor in the philosophical principle of unicentrism and in the concept of a systems transdisciplinary approach is played by the universal order. This order, which manifests itself in fragments of space, attributes of information and periods of time, ensures the inevitable achievement of a certain result by a certain functional ensemble of objects, in a certain place and at a certain time.

Thus, the binding of the content of a system-forming factor to a specific philosophical principle, the description of this factor within the framework of the concept of a specific type of systems approach has a fascinating perspective. This is an exciting prospect because it entails not only the discovery of new ways to understand, design, engineer or govern systems, 
but it means that General Systemology, informed by General Systems Theory, will reveal systemic structures and mechanisms unknown to and unanticipated by contemporary science. Progress towards a General Systems Theory will therefore not only unify the systems field but initiate an important cycle of scientific discovery (Rousseau, Billingham, Wilby, Blachfellner, 2016 b).

Currently, the development of a general theory of systems has received a new impetus thanks to the initiative of the members of the Research Group Systems Science and Philosophy. This group is part of the Bertalanffy Center for the Study of Systems Science (Austria). In August 2015, at the annual conference of the International Society of Systems Sciences, members of the group published a Manifesto for General Systems Transdisciplinarity. In this Manifesto, they discussed the motivation to implement this project, gave an overview of the key studies they conducted to ensure the possibility and importance of creating such a transdiscipline (Rousseau, Wilby, Billingham, Blachfellner, $2016 \mathrm{c}$ ). Within the framework of the classification of system approaches, it was possible to identify a claimant for the role of such a transdiscipline. Such a challenger is a systems transdisciplinary approach. The philosophical foundations, the concept and methodology of the systems transdisciplinary approach have been developed by specialists of the Russian School of Transdisciplinarity and the Institute of Transdisciplinary Technologies since 1990 (http://td-science.ru/index.php/history).

\section{References:}

1. Anokhin, P. (1975). Essays on Physiology of Functional Systems. Medicina, Moscow, p. 25.

2. Bertalanffy, L. von. (1967). General Theory of Systems: Application to Psychology. Social Science. Information sur les Sciences Sociales, vol. VI, 6, p. 125-136.

3. Hall M.L.W. (1995). Merging the World Views of Systems Science and Human Values. In Critical Issues in Systems Theory and Practice; Springer, Boston, pp. 131-135.

4. Knyazeva, E. (2011). Transdisciplinary Research Strategies. Vestnik TGPU, 10 (112), pp. 193-201.

5. Mokiy, V. (2009). Foundations of Transdisciplinarity. El-Fa, Nalchik, pp. 29-30.

6. Mokiy, V. Lukyanova, T. (2017). Methodology of Scientific Research. Transdisciplinary Approaches and Methods. Yurayt, Moscow, p. 46.

7. Nikiforov, A. (2010). The New Philosophical Encyclopedia. In four volumes. vol. IV. Thought, Moscow, pp. 299-300.

8. Petts, J. Owens, S. Bulkeley, H. (2008). Crossing Boundaries: Interdisciplinarity in the Context of Urban Environments. Geoforum, 39 (2), pp. 593-601. 
9. Porus, V. (1995) The System Meaning of the Concept "Scientific Rationality": Rationality as a Subject of Philosophical Research. Institute Philosophe RAS, Moscow, p. 85.

10. Rousseau, D. Billingham, J. Wilby, J. Blachfellner, S. (2016 a). In Search of General Systems Theory. Systema. Special Issue - General Systems Transdisciplinarity, 4(1); pp. 76-99.

11. Rousseau, D. Billingham, J. Wilby, J. Blachfellner, S. (2016 b). The Synergy between General Systems Theory and the General Systems Worldview. Systema 4(1) Special Issue - General Systems Transdisciplinarity, pp. 61-75.

12. Rousseau, D. Wilby, J. Billingham, J. Blachfellner, S. (2016 c). Manifesto for General Systems Transdisciplinarity. Systema 4(1), Special Issue - General Systems Transdisciplinarity: pp. 4-14.

13. Rousseau, D. Billingham, J. (2018). A Systematic Framework for Exploring Worldviews and Its Generalization as a Multi-Purpose Inquiry Framework. Systems, Vol.6, Issue 3, 27.

14. Sadovcky, V. (1974). Bases of the General Theory of Systems. Nauka, Moscow, p. 238. 